\title{
Contributions of Striatal Subregions to Place and Response Learning
}

\author{
Henry H. Yin ${ }^{1}$ and Barbara J. Knowlton \\ Department of Psychology, University of California, Los Angeles, Los Angeles, California 90095-1563, USA
}

\begin{abstract}
The involvement of different subregions of the striatum in place and response learning was examined using a T-maze. Rats were given NMDA lesions of the dorsolateral striatum (DLS), anterior dorsomedial striatum (ADMS), posterior dorsomedial striatum (PDMS), or sham surgery. They were then trained to retrieve food from the west arm of the maze, starting from the south arm, by turning left at the choice point. After $7 \mathrm{~d}$ of training, with four trials a day, a probe test was given in which the starting arm is inserted as the north arm, at the opposite side of the maze. A left turn would indicate a "response" strategy; a right turn, a "place" strategy. The rats were then trained for 7 more days, followed by a second probe test. Unlike rats in the other groups, most of the rats in the PDMS group turned left, using the response strategy on both probe tests. These results suggest that the PDMS plays a role in spatially guided behavior.
\end{abstract}

When a rat is trained to run down a T-maze and retrieve food from one of the arms, it may have learned to make a particular turn response, or it may have learned that the food is at a particular place (Restle 1957). In normal animals, there is a shift from a place strategy to a response strategy in the course of training (Packard 1999). Early in training, behavior appears to be determined by the integration of the environmental cues with the location of the goal, as rats tend to enter the arm that had been baited even when starting from a different arm, thus responding flexibly and not simply with a particular turning response. With extended training, however, behavior becomes more inflexible and response-specific: Rats tend to make the same turning response, regardless of which arm they start from. Although the basis for responding shifts with extended training, it appears that place learning and response learning occur in parallel, and can be dissociated in terms of their neural substrates. For example, rats with inactivation of the neostriatum (dorsal striatum) use predominantly a place strategy, whereas after inactivation of the hippocampus rats show predominantly a response strategy (Packard and McGaugh 1996).

These important observations led to the idea that the hippocampus and the neostriatum belong to different neural systems which mediate place and response learning, respectively, and that disrupting neural activity in either structure results in the compensatory action of the other system in controlling behavior (Packard and Knowlton 2002).

Thus, previous research suggested a dissociation between the hippocampus-dependent place system for flexible behavior based on integration of contextual cues, and the neostriatumdependent response system for inflexible habits based on stimulus-response (S-R) associations. But it has also been noted that the neostriatum appears to be a highly heterogeneous structure (Devan and White 1999; Devan et al. 1999). Given the large size of this structure, the regional differences in anatomical connections with other neural structures, and the diverse profiles of neural activity in behaving animals, it seems likely that different neostriatal regions support different behavioral functions. Devan et al. (1999), for example, found that lesions of the dorsomedial

\section{'Corresponding author.}

E-MAIL hyin@ucla.edu; FAX (310) 206-5895.

Article and publication are at http://www.learnmem.org/cgi/doi/10.1101/ Im.81004. striatum (DMS) disrupted some aspects of spatial learning, resulting in a preference for the cue strategy (visible platform) in the water maze and increased swimming around the periphery of the pool. This finding contrasted with the effect of lesions of the dorsolateral striatum (DLS), which did not affect learning to locate a hidden platform on the basis of spatial cues, but rather decreased the use of the cue strategy. Because, however, the lesions in this study were electrolytic, it is not clear whether the effects were mediated by the destruction of fibers of passage, rather than cells within the DMS. On the other hand, others have found that the DMS is involved in conditional S-R learning (Adams et al. 2001). After reviewing the available studies, therefore, Packard and Knowlton (2002) concluded that there was as yet no definitive evidence for a selective role of the DMS in the type of spatial and contextual learning characteristic of the hippocampus.

One possible reason for these mixed results is that the DMS itself is heterogeneous, and only a specific region within it plays a role in spatial learning. If evidence of dorsomedial striatal involvement in spatial learning could be found, it would suggest that separate regions of the dorsal striatum play very different roles in mediating behavior-a view supported by available anatomical evidence. All cortical areas, including the hippocampus, project to the striatum (McGeorge and Faull 1987, 1989; Parent 1990; Berendse et al. 1992). The neostriatum receives two major classes of cortical inputs. On the one hand, the primary sensorimotor cortices project massively to the DLS, or sensorimotor striatum. On the other, the projections to the DMS come from association cortices generally. Within this latter class of projections, again two groups of projections could be discerned: the anterior region receives projections mainly from the prefrontal cortex, whereas the more posterior region receives, in addition, significant projections from the perirhinal and agranular insular regions, as well as the entorhinal cortex and basolateral amygdala (McGeorge and Faull 1987, 1989).

Based on these observations, in the present study we assessed the effects of selective, excitotoxic lesions of different striatal subregions on place and response learning using a $\mathrm{T}$ maze. In particular, to assess the role of the DMS in place learning, we tested whether the anterior dorsomedial striatum (ADMS) and the posterior dorsomedial striatum (PDMS) could be dissociated functionally. 


\section{RESULTS}

Figure 1 provides schematic representation of the NMDA lesions (Paxinos and Watson 1998). Inspection of the stained tissue did not reveal extensive damage outside of the striatum for the DLS, PDMS, or ADMS lesions. Cell loss and gliosis were seen in the targeted striatal area. Tissue shrinkage resulted in a visible widening of the lateral ventricles in all cases.

Acquisition data are illustrated in Figure 2. Rats in the lesioned groups and the sham group learned to go to the reinforced arm of the T-maze accurately over the course of training. There was a significant improvement in choice accuracy $\left(F_{13,585}=28.4, P<0.01\right)$, but no main effect of lesion $(F<1)$ nor any interaction between lesion type and extent of training $(F=1.2)$.

Moreover, there was a general reduction in choice latency over days $\left(F_{13,585}=29.74, P<0.0001\right)$. Although the lesioned groups were as accurate as rats in the sham-lesioned group, they responded more slowly. There was a significant effect of lesion $\left(F_{3,45}=4.1\right.$, $P<0.05)$, and a significant interaction between lesion type and days $\left(F_{39,585}=3.1, P<0.01\right)$. The PDMS and DLS groups were significantly slower than the other two groups at first, but the difference gradually disappeared over the course of training, and all groups were choosing the correct arm with very short latency during the second week of training.

Data from the first probe test are shown in Figure 3. Compared to the sham group, rats in the PDMS group predominantly displayed a response strategy on the first probe test with the start arm rotated $180^{\circ}\left(\chi^{2}{ }_{1,26}=5.38, P<0.05\right)$. The majority of PDMS rats did not enter the arm in the same location as the trained arm, but rather made the same left turn response that was reinforced during training. In contrast, rats in the sham group were about evenly divided between responding based on location and responding based on response. Rats in the ADMS group performed similarly to rats in the sham group. Although rats in the DLS group used the place strategy more frequently (7 out of 9 rats), this pattern was not significantly different from the performance of rats in the sham group $\left(\chi_{1,20}^{2}=2.15, P>0.05\right)$.

On the second probe test (Fig. 3), rats in the sham group showed numerically more response-based choices and were no longer significantly different from the PDMS group $\left(\chi_{1,26}^{2}=2.07\right.$, $P>0.05)$. The ADMS and DLS groups did not differ significantly from the sham group, although the DLS group continued to show a numerically greater tendency to respond based on the location of the trained arm.

\section{DISCUSSION}

Through the use of probe tests on a simple T-maze task, we demonstrated that lesions of the PDMS increased the proportion of rats using a response rather than a place strategy early in training. This pattern was not evident when the lesion placement was more anterior (ADMS). However, because the placement of PDMS lesions was close to the lateral septal nucleus and the lateral ventricles, it is possible that extra-striatal damage may have resulted. In particular, diffusion of NMDA to the nearby lateral septal nucleus may have been responsible for the observed deficits in place learning. Although we did not discover any cell loss or gliosis in our histological examination of the septum, minor damage cannot be ruled out. Lesions of the DLS, on the other hand, resulted in a numerically more frequent use of the place strategy; though this effect did not reach statistical significance, it is consistent with previous findings (Packard and McGaugh 1996).

Previous studies have provided conflicting data on the role of the DMS, also known as associative striatum, in place learning (for review, see Packard and Knowlton 2002). Using more selective lesions in the present study, we showed that rats with PDMS damage rarely used the place strategy on the probe test, whereas ADMS damage had no effect on probe test behavior. It is not clear, however, whether the PDMS animals were unable to use a place strategy per se or were simply biased to acquire a response strategy. According to the first possibility, PDMS rats may have a deficit in spatial behavior and are thus only able to acquire the response strategy. In contrast, according to the second possibility PDMS rats could simply be neglecting spatial cues because acquisition of a response strategy is facilitated. Under some conditions (e.g., when the spatial cues are made more prominent), the PDMS rats may use the place strategy more frequently. Further work is 
A

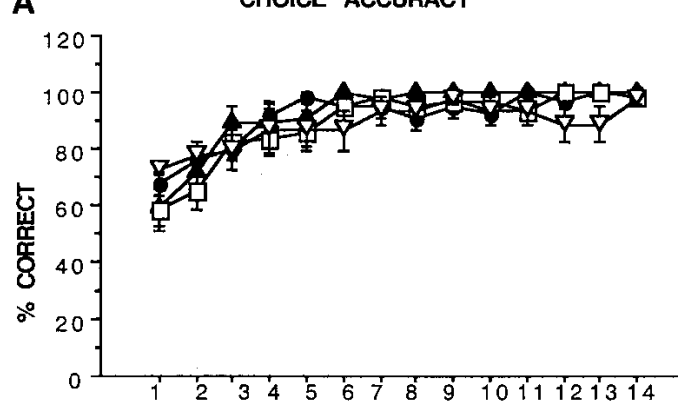

B

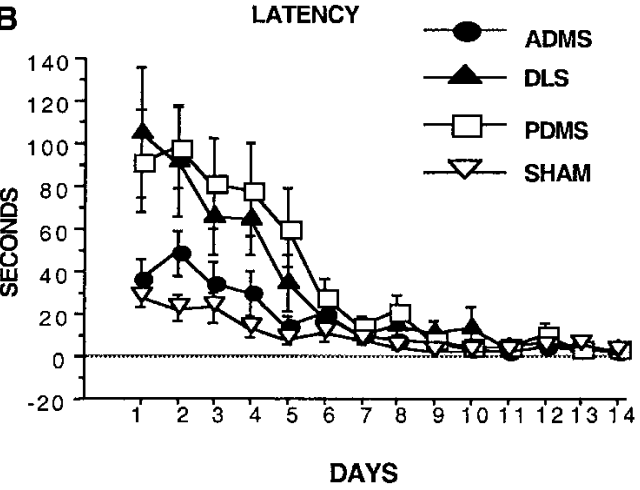

Figure 2 Performance across $14 \mathrm{~d}$ of acquisition training. Abbreviations as in Figure 1.

required to determine whether the place strategy deficit observed here in the PDMS rats reflects a general impairment in spatial learning.

In the present study, relatively few visual cues were available in the maze environment compared to many of the previous studies of spatial learning. It has been known for many decades that the nature of the spatial environment influences the choice of "place" or "response" strategies (Restle 1957). Our choice of a relatively cue-deprived environment would accordingly lead to a greater number of animals using the response strategy. Indeed, on the first probe test, control animals were almost evenly divided between those displaying place and response strategies. This gave us the opportunity to see increases in either the place or response strategy in the experimental groups. However, as noted above, it is possible that PDMS rats would use a place strategy if more spatial cues were provided. It may be important to compare the effects of PDMS lesions and hippocampal lesions, which impair place learning even when spatial cues are abundant.

Much evidence suggests that the neostriatum mediates response learning, whereas the hippocampus mediates place learning. This claim is based on an extensive series of studies using various mazes in addition to the place/response procedure employed in the present study (for review, see Packard and Knowlton 2002). The general logic behind these studies is to control the motivational and motor requirements while dissociating different types of learning. For example, the win-shift task on the radial-arm maze, in which animals must remember previously visited arms to determine the correct choice, is a test of flexible spatial learning. The win-stay task, on the other hand, requires rats to approach the arms based on a discrete cue, thus providing a measure of S-R learning (Packard et al. 1989; Sage and Knowlton 2000). Lesions of the hippocampus impair win-shift learning, whereas lesions of the neostriatum impair win-stay learning (McDonald and White 1993). Despite differences in procedure, a similar dissociation between flexible spatial learning and inflexible S-R learning has been found using the water maze as well as the cross maze (Packard et al. 1989; Packard and McGaugh 1992; McDonald and White 1993; Devan and White 1999; Devan et al. 1999). The most striking demonstration of this dissociation using the place/response task can be found in a study by Packard and McGaugh (1996). Those investigators inactivated either the dorsal striatum or the hippocampus with lidocaine just before probe tests, to assess the relative contributions of these two brain regions to the expression of previously learned behavior. After $7 \mathrm{~d}$ of training on the maze, control rats and rats with striatal infusions used predominantly a place strategy. After additional training, controls mostly shifted to the use of the response strategy, whereas the animals with striatal inactivation exhibited the use of place strategy. Inactivation of the hippocampus, in contrast, selectively impaired the expression of place learning (Packard and McGaugh 1996). In light of such results, Packard and Knowlton (2002) concluded that "the basal ganglia and the hippocampus are parts of independent memory systems that mediate the acquisition of S-R habits and cognitive (e.g., Tolman 1932) forms of memory, respectively."

This view, however, is in need of revision, both on grounds of the anatomical connectivity and in light of the present findings of heterogeneous effects of lesions within the neostriatum. The hippocampus and neostriatum have historically been assumed to belong to two functionally distinct systems (Mishkin et al. 1984). Histologically, the laminated hippocampus, with glutamatergic projection cells, is a type of primitive cortex, and the unlaminated neostriatum, with GABAergic projection cells, belongs to the subcortical, or basal, nuclei of the cerebrum. The distinction between the two structures, in this sense, appears to hold insofar as the cerebral cortex in general is to be distinguished from the cerebral nuclei (Swanson 2000). Functionally, however, the crucial consideration must be given to the connections of these structures; and here a rather different picture

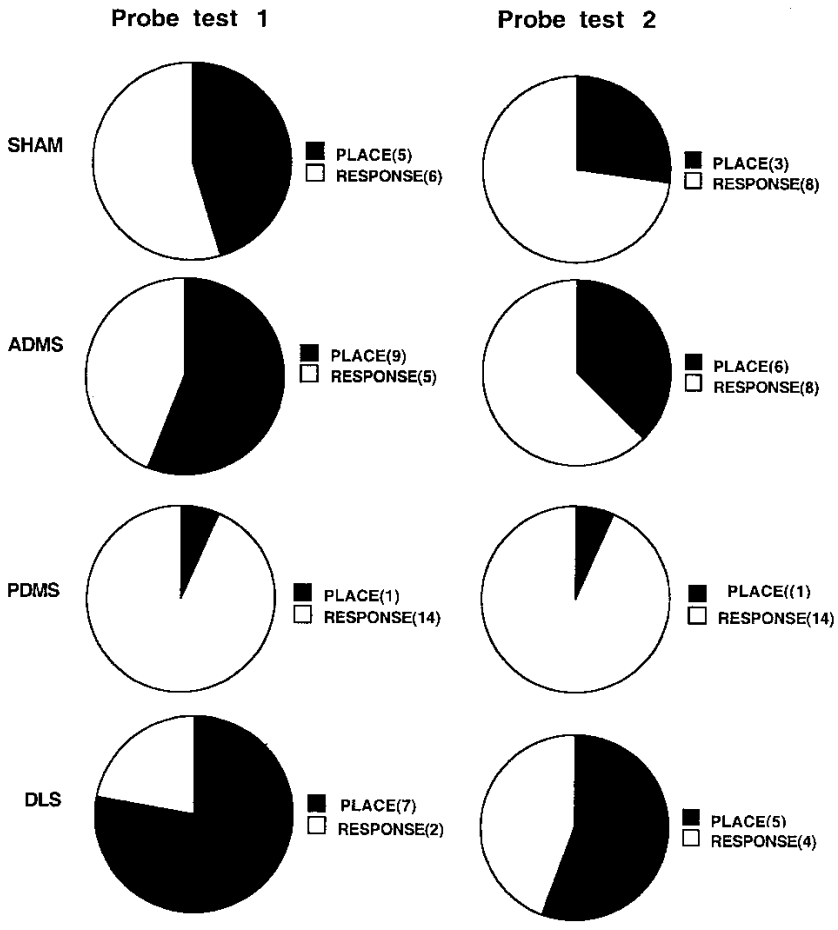

Figure 3 The results from both probe tests. Abbreviations as in Figure 1. The numbers in the parentheses show how many rats from each lesion group made that particular choice (place or response). 
emerges. Whereas the major projections from the hippocampus proper to the subcortical cerebral nuclei are directed to the lateral septal nucleus, the remaining parts of the hippocampal formation, such as the entorhinal cortex, send significant projections to the PDMS. In short, hippocampal regions known to be involved in place learning are also strongly connected with certain striatal regions, particularly the PDMS (McGeorge and Faull 1989).

A number of investigators have noticed such differences in connectivity, and conducted studies to test the hypothesis that the DMS is also involved in behavioral flexibility and spatial learning (Devan and White 1999; Devan et al. 1999; Sakamoto and Okaichi 2001; Ragozzino 2003). For example, on a hiddenplatform water maze task, lesions of the DMS impaired the use of distal spatial cues in navigation, resulting in a preference for use of S-R memory when competing place- and cue-learning preferences were simultaneously assessed (Whishaw et al. 1987; Devan and White 1999). After lesions of the DMS, rats can use place strategies for spatial navigation, but if the response solution is available they ignore place information and use the response strategy instead (Whishaw et al. 1987). Such findings conflict with the claim that the neostriatum as a whole is involved in S-R learning per se, in contrast to the hippocampus. Rather, they suggest that the functions of the DMS are closely related to those of the hippocampus in spatial navigation.

Our results suggest that the mixed results in the literature can be attributed, at least in part, to the placement of lesions. A particularly important observation from the present study is that there are two functionally dissociable regions along the anteriorposterior axis of the rat neostriatum. Whereas the PDMS appears to be critical for the use of the place strategy, the ADMS is not. Because previous work did not take into account this anteriorposterior distinction, the mixed results may have been due to lesion placement. A more posterior placement in the DMS, as our data suggest, would lead to deficits in the use of the place strategy. Thus previous studies that produced large DMS lesions damaging the PDMS would be expected to produce deficits in responding based on spatial cues. This conclusion is in accord with earlier findings indicating a preference for the response strategy when given a choice after DMS lesions (Whishaw et al. 1987; Devan and White 1999).

On the basis of the present results as well as results from other studies (Whishaw et al. 1987; Devan and White 1999; Devan et al. 1999; Sakamoto and Okaichi 2001), we propose that both the hippocampal formation and the PDMS are components of a larger corticostriatal system mediating flexible, goal-directed responding based on an integrated representation of the environment. This view is an extension of the widely accepted claim that, in the cerebral hemispheres, the functional unit capable of integrated behavioral functions is neither a part of cortex nor a part of striatum, but rather a corticobasal ganglia circuit (Alexander et al. 1986; Alexander and Crutcher 1990). Rather than the contrast between hippocampus and striatum proposed by previous researchers, we suggest that the contrast is between two or more corticobasal ganglia circuits. This conclusion has important implications for future research. For example, researchers have long been interested in how such memory systems may interact, and numerous studies have attempted to find possible connections between the hippocampus and striatum (Poldrack and Packard 2003). But if the interaction is between multiple corticobasal ganglia circuits, then there could be a number of levels at which functional interaction is possible (Joel and Weiner 1994; Haber 2003).

However, although the hippocampal formation and the PDMS may belong to the same functional system, it is unlikely that they play identical roles within this system. Although there is extensive evidence for the role of the hippocampal formation in spatial navigation, the role of the PDMS is less clear. The PDMS is uniquely positioned to incorporate extensive inputs from the hippocampal formation and medial prefrontal regions which have been shown to be involved in goal-directed action (Corbit and Balleine 2003). Thus, the role of the PDMS may not be spatial processing per se, but rather the integration of spatial information in the environment for the purpose of reaching a desired goal.

\section{MATERIALS AND METHODS}

\section{Subjects}

Forty-nine male Long-Evans rats (350-500 g) were used. They were bred in the UCLA Psychology Department vivarium from Harlan stock, and individually housed on a $14 \mathrm{~h}: 10 \mathrm{~h}$ light:dark cycle, with unrestricted access to water. Prior to this study they were used in a study of instrumental conditioning, in which they learned to press two levers for sucrose solution and pellets. They were food-deprived throughout this period, and maintained at about $80 \%$ of their normal weight by receiving $10-15 \mathrm{~g}$ of home chow after training each day.

Rats were randomly assigned to four groups: Sham $(n=11)$, DLS $(n=9)$, ADMS $(n=14)$, PDMS $(n=15)$. They were anesthetized with sodium pentobarbital (Nembutal; $50 \mathrm{mg} / \mathrm{kg}$ ), treated with atropine $(0.1 \mathrm{mg})$, and placed in a stereotaxic instrument. Small holes were drilled into the skull bilaterally, and 28-gauge cannulae were lowered into the brain at the following coordinates: DLS, $0.7 \mathrm{~mm}$ anterior to bregma and $3.6 \mathrm{~mm}$ lateral to midline, and $5 \mathrm{~mm}$ below the skull surface; ADMS, $1.2 \mathrm{~mm}$ anterior and $1.7 \mathrm{~mm}$ lateral to bregma, and $5 \mathrm{~mm}$ below skull surface; PDMS, $0.4 \mathrm{~mm}$ posterior and $2.6 \mathrm{~mm}$ lateral to bregma, and $4.5 \mathrm{~mm}$ below skull surface. For the lesion groups, $0.4 \mu \mathrm{L}$ of NMDA per side $(20 \mathrm{mg} / \mathrm{mL}$; Sigma) was infused over $3 \mathrm{~min}$; for the sham group, no infusion was given. Three minutes after the infusion, the cannulae were removed.

At the end of the experiment, the rats were sacrificed using a lethal barbiturate overdose and perfused transcardially with $0.9 \%$ saline followed by $10 \%$ formaldehyde solution. The brains were stored in a $25 \%$ sucrose-formalin solution for at least $3 \mathrm{~d}$ before $50 \mu \mathrm{m}$ coronal sections were cut throughout the anterior striatum. The slices were stained with thionin and examined.

\section{Apparatus}

The apparatus was a T-maze with removable arms made of wood and covered in white laminate, surrounded by black curtains extending from $30 \mathrm{~cm}$ above the floor and $50 \mathrm{~cm}$ below the ceiling, and located in a dimly lit and quiet room. It was elevated $64 \mathrm{~cm}$ from the floor. Its center platform was $91 \mathrm{~cm}$ in diameter, and each arm was $69 \mathrm{~cm}$ in length and $10 \mathrm{~cm}$ in width, with a recessed food well at the end of each arm. Extra-maze cues were primarily limited to the light from an open door at the south side of the testing room. The experimenter stood in the maze room at a consistent location just outside of the curtains to observe the rats.

\section{Training}

Throughout this study the experimenters performing behavioral testing were blind to the group assignment. The day before training began, all rats were acclimated to the maze for $5 \mathrm{~min}$, during which they were allowed to traverse the entire maze freely. The training consisted of allowing the rats to visit the food cup to retrieve a piece of Apple Jack cereal at the end of the west arm. The arm it entered, east or west, was recorded as soon as the entire body, including the tail, was in the arm. At the same time a stopwatch was used to record the latency in sec. On each day of the training phase, four trials were given in a row to every rat. The rat was removed from the maze as soon as it consumed the Apple Jack, or after $300 \mathrm{sec}$. Between each trial the rat was placed in an opaque holding box behind the starting arm for $\sim 10$ sec. 
The maze was cleaned frequently throughout the session to minimize odor cues. The 49 rats in the study were tested in the same order each day. Members of the four lesion groups (DLS, ADMS, PDMS, and SHAM) were equally distributed throughout the testing order.

After $7 \mathrm{~d}$ of training, a probe test was given in which the originally south arm was inserted as the north arm and used as the starting arm. No food was present in the maze during this test, and choice and latency were also recorded as in training. Following the first probe test on day 8 , another $7 \mathrm{~d}$ of training and another probe test were given, followed by a second probe test.

\section{ACKNOWLEDGMENTS}

We thank Denise Yanez and Erica Espinoza for their excellent technical support. This research was supported by an NSF graduate fellowship to H.H.Y. and an NSF grant \#9985417 to B.J.K.

The publication costs of this article were defrayed in part by payment of page charges. This article must therefore be hereby marked "advertisement" in accordance with 18 USC section 1734 solely to indicate this fact.

\section{REFERENCES}

Adams, S., Kesner, R.P., and Ragozzino, M.E. 2001. Role of the medial and lateral caudate-putamen in mediating an auditory conditional response association. Neurobiol. Learn. Mem. 76: 106-116.

Alexander, G.E. and Crutcher, M.D. 1990. Functional architecture of basal ganglia circuits: Neural substrates of parallel processing. Trends Neurosci. 13: 266-271.

Alexander, G.E., DeLong, M.R., and Strick, P.L. 1986. Parallel organization of functionally segregated circuits linking basal ganglia and cortex. Annu. Rev. Neurosci. 9: 357-381.

Berendse, H.W., Galis-de Graaf, Y., and Groenewegen, H.J. 1992. Topographical organization and relationship with ventral striatal compartments of prefrontal corticostriatal projections in the rat. $J$. Comp. Neurol. 316: 314-347.

Corbit, L.H. and Balleine, B.W. 2003. The role of prelimbic cortex in instrumental conditioning. Behav. Brain Res. 146: 145-157.

Devan, B.D. and White, N.M. 1999. Parallel information processing in the dorsal striatum: Relation to hippocampal function. J. Neurosci. 19: 2789-2798.

Devan, B.D., McDonald, R.J., and White, N.M. 1999. Effects of medial and lateral caudate-putamen lesions on place- and cue-guided behaviors in the water maze: Relation to thigmotaxis. Behav. Brain Res. 100: 5-14.

Haber, S.N. 2003. The primate basal ganglia: Parallel and integrative networks. J. Chem. Neuroanat. 26: 317-330.

Joel, D. and Weiner, I. 1994. The organization of the basal ganglia-thalamocortical circuits: Open interconnected rather than closed segregated. Neuroscience 63: 363-379.
McDonald, R.J. and White, N.M. 1993. A triple dissociation of memory systems: Hippocampus, amygdala, and dorsal striatum. Behav. Neurosci. 107: 3-22.

McGeorge, A.J. and Faull, R.L. 1987. The organization and collateralization of corticostriate neurones in the motor and sensory cortex of the rat brain. Brain Res. 423: 318-324.

McGeorge, A.J. and Faull, R.L. 1989. The organization of the projection from the cerebral cortex to the striatum in the rat. Neuroscience 29: 503-537.

Mishkin, M., Malamut, B., and Bachevalier, J. 1984. Memories and habits: Two neural systems. In Neurobiology of learning and memory (eds. G. Lynch et al.), pp. 65-77. Guilford Press, New York.

Packard, M.G. 1999. Glutamate infused posttraining into the hippocampus or caudate-putamen differentially strengthens place and response learning. Proc. Natl. Acad. Sci. 96: 12881-12886.

Packard, M.G. and Knowlton, B.J. 2002. Learning and memory functions of the basal ganglia. Annu. Rev. Neurosci. 25: 563-593.

Packard, M.G. and McGaugh, J.L. 1992. Double dissociation of fornix and caudate nucleus lesions on acquisition of two water maze tasks: Further evidence for multiple memory systems. Behav. Neurosci. 106: 439-446.

Packard, M.G. and McGaugh, J.L. 1996. Inactivation of hippocampus or caudate nucleus with lidocaine differentially affects expression of place and response learning. Neurobiol. Learn. Mem. 65: 65-72.

Packard, M.G., Hirsh, R., and White, N.M. 1989. Differential effects of fornix and caudate nucleus lesions on two radial maze tasks: Evidence for multiple memory systems. J. Neurosci. 9: 1465-1472.

Parent, A. 1990. Extrinsic connections of the basal ganglia. Trends Neurosci. 13: 254-258.

Paxinos, G. and Watson, C. 1998. The rat brain in stereotaxic coordinates. Academic Press, San Diego, CA

Poldrack, R.A. and Packard, M.G. 2003. Competition among multiple memory systems: Converging evidence from animal and human brain studies. Neuropsychologia 41: 245-251.

Ragozzino, M.E. 2003. Acetylcholine actions in the dorsomedial striatum support the flexible shifting of response patterns. Neurobiol. Learn. Mem. 80: 257-267.

Restle, F. 1957. Discrimination of cues in mazes: A resolution of the "place-vs.-response" question. Psychological Rev. 64: 217.

Sage, J.R. and Knowlton, B.J. 2000. Effects of US devaluation on win-stay and win-shift radial maze performance in rats. Behav. Neurosci. 114: 295-306.

Sakamoto, T. and Okaichi, H. 2001. Use of win-stay and win-shift strategies in place and cue tasks by medial caudate putamen (MCPu) lesioned rats. Neurobiol. Learn. Mem. 76: 192-208.

Swanson, L.W. 2000. Cerebral hemisphere regulation of motivated behavior. Brain Res. 886: 113-164.

Whishaw, I.Q., Mittleman, G., Bunch, S.T., and Dunnett, S.B. 1987. Impairments in the acquisition, retention and selection of spatial navigation strategies after medial caudate-putamen lesions in rats. Behav. Brain Res. 24: 125-138.

Received September 22, 2003; accepted in revised form June 9, 2004. 


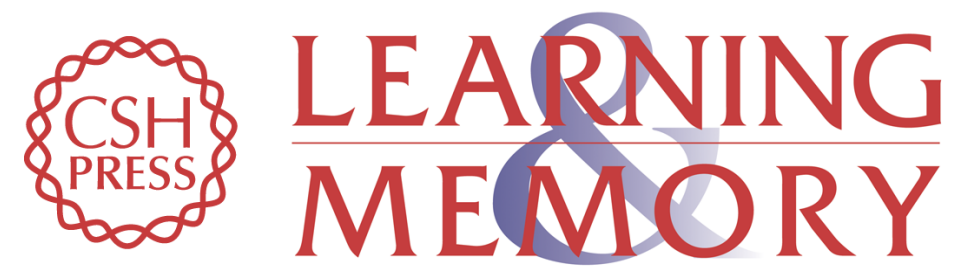

\section{Contributions of Striatal Subregions to Place and Response Learning}

Henry $\mathrm{H}$. Yin and Barbara J. Knowlton

Learn. Mem. 2004, 11:

Access the most recent version at doi:10.1101//m.81004

References This article cites 24 articles, 3 of which can be accessed free at: http://learnmem.cshlp.org/content/11/4/459.full.html\#ref-list-1

License

Email Alerting

Receive free email alerts when new articles cite this article - sign up in the box at the Service top right corner of the article or click here. 